\title{
Aurora kinases: A novel target for drug development
}

\section{Liana Dedina}

University of Toronto

In recent years, a novel hypothesis in cancer therapeutics has been proposed. It states that abnormal expression of aurora kinases (AURK) contributes to neoplastic transformation and carcinogenesis, and that inhibitors of AURK can provide a valuable tool in the chemotherapeutic arsenal. As such, AURK inhibitors have become a hot item on Big Pharma's list. So far, preclinical and clinical data point towards the inhibitors' activity against solid tumors, with mainly cytostatic effects on cancer stabilization. There is great hope in the eventual implementation of these drugs in clinical practice.

AURK are a family of highly conserved serine-threonine kinases $^{1,2}$, both in structure and in function ${ }^{3}$. AURK consist of three members: AURK-A, AURK-B, and AURK-C ${ }^{1}$. AURK-A is expressed in most human cells ${ }^{1}$. It is involved in the regulation of key cellular events that take place during mitosis: centrosomal function, bipolar spindle assembly and $\mathrm{G}_{2}-\mathrm{M}$ transition ${ }^{1,4}$. AURK-B is ubiquitously expressed and contributes to chromatin modification, chromatid segregation and cytokinesis ${ }^{2}$. Functions of AURK-C are limited to spermatogenesis, and thus do not play a crucial role in cancer development.

Given their physiological functions, it is not surprising that deregulation of AURK-A and AURK-B is associated with tumourigenesis ${ }^{1}$. AURK-A's role in tumor development is currently the most well-defined among AURKs ${ }^{1}$. Overexpression of AURK-A is observed in colon, breast, pancreas, liver and bladder cancers ${ }^{4}$. This may arise due to gene amplification or post-translational modifications ${ }^{2}$. Moreover, overexpression of AURK-A in hepatocellular carcinoma has been shown to correlate with both the stage and grade of tumor ${ }^{5}$. In addition, AURK-A has been shown in vitro to enable the production of multipolar spindles, resulting in genomic instability ${ }^{2}$ in fibroblast cell cultures. However, the AURK-A gene is not established as an oncogene due to inconsistent findings in the literature $^{2,4}$. Given that AURK-A alone may not lead to tumourigenesis, interactions with other proteins, such as tumor suppressors, may be of importance. The interactions between AURK-A and the tumor suppressor p53, a protein involved in preventing cancer, have already been well characterized ${ }^{24}$. AURK-A can phosphorylate p53 at two sites: 1) Ser-215 phosphorylation prevents activation of p53 downstream targets $^{6}$; 2) Ser-315 phosphorylation facilitates p53 protein degradation ${ }^{7}$. Taken together, these phosphorylation events may desensitize cells for apoptosis ${ }^{1}$. Moreover, activation of $\mathrm{G}_{1}$ checkpoint depends on p53 status ${ }^{4}$. Therefore, AURK-A-induced suppression of p53 activity may allow aneuploid cells to progress through the cell cycle ${ }^{1,2}$. Overall, the data suggest that AURK-A, along with other factors, may play a role in promoting carcinogenesis ${ }^{2}$.

The precise role of AURK-B in cancer development, however, is not nearly as clear ${ }^{1}$. Several human tumors have been observed to overexpress the enzyme ${ }^{2}$, including lung, prostate, kidney, breast and colorectal tumors ${ }^{4}$. In particular, a positive correlation between AURK-B expression and the stage of primary colorectal cancer has also been reported ${ }^{8}$. These results were also associated with poor prognosis in patients with higher AURK-B levels ${ }^{8}$. Similar findings were reported in patients with endometrial carcinoma ${ }^{9}$. Therefore, it stands to reason that AURK-B, along with AURK-A, may also be involved in multiple pathways leading to carcinogenesis.

Uncontrolled cellular growth is one of the main characteristics of cancer ${ }^{4}$. Consequently, suppression of cellular division provides a means for therapeutic 
intervention and treatment of multiple cancer types ${ }^{1,2}$. The overexpression of AURK in select tumor types, along with its associations with genetic instability and regulation of mitotic events make these enzymes an attractive target for drug development ${ }^{1,4}$. The potential of AURK as a drug target was demonstrated in RNA interference experiments, where gene-silencing in human cells lead to suppression of tumor growth and increased sensitivity to chemotherapy ${ }^{2}$. Currently, there are a number of AURK inhibitors at different stages of development ${ }^{1}$. AURK inhibitors may be used in combination with other available chemotherapies. For instance, doxorubicin treatment of prostate cancer cells was shown to be more effective when treated concurrently with MK0457, an AURK inhibitor ${ }^{4}$.

The significance of AURK inhibitors in a clinical setting has yet to be determined. Ongoing phase I trials are faced with several challenges. First, the optimization of drug administration to patients in order to maximize AURK inhibition and exert minimal toxicological consequences needs to be carefully characterized. Second, there is currently no predictive biomarker to identify and select patients for AURK inhibitor treatment. Finally, despite numerous pre-clinical trials, synergistic and additive anti-cancer effects of AURKinhibitors and existing chemotherapies have yet to be translated into clinical practice.

So, are AURK inhibitors really the 'it drugs' of the future? As with any new chemotherapeutic agent, there are a few uncertainties associated with clinical utility of AURK inhibitors. First, the involvement of pharmacogenetic and environmental factors in drug effectiveness are not defined. Second, implications of inter-individual response variability are still unknown. Lastly, the long-term effects of treatment have yet to be determined. Nonetheless, AURK inhibitors are promising, given their roles in regulation of the cell cycle. Successful clinical implementation of AURK inhibitors will bring us a step closer to the development of superior cancer treatment.

The finish line is almost in sight, and the race to conquer cancer continues.

\section{References}

1. Dar AA, Goff LW, Majid S, Berlin J, El-Rafai W. Aurora kinase inhibitors - rising stars in cancer therapeutics? Mol Cancer Ther 2010;9(2):268-278.

2. Fu J, Bian M, Jiang Q, Zhang C. Roles of aurora kinases in mitosis and tumourigenesis. Mol Cancer Res 2007;5(1):110 .
3. Adams R, Carmena M, Earnshaw W. Chromosomal passengers and the (aurora) ABCs of mitosis. Trends Cell Biol 2001;11(2):49-54.

4. Lok W, Klein RQ, Saif MW. Aurora kinase inhibitors as anti-cancer therapy. Anti-Cancer Drugs 2010;21(4):339350.

5. Jeng YM, Peng SY, Lin CY, Hsu HC. Overexpression and amplification of Aurora-A in hepatocellular carcinoma. Clin Cancer Res 2004;10(6):2065-2071.

6. Liu Q, Kaneko S, Yang L, et al. Aurora-A abrogation of p53 DNA binding and transactivation activity by phosphorylation of serine 215. J Biol Chem 2004;279(50):52175 - 52182.

7. Katayama H, Sasai K, Kawai H, et al. Phosphorylation by aurora kinase A induces Mdm2-mediated destabilization and inhibition of p53. Nat Genet 2004;36(1):55 - 62 .

8. Katayama H, Ota $\mathrm{T}$, Jisaki $\mathrm{F}$, et al. Mitotic kinase expression and colorectal cancer progression. J Natl Cancer Inst 1999;91(13):1160-1162.

9. Kurai M, Shiozawa T, Shih H, Miyamoto T, Feng YZ, Kashima H, et al. Expression of Aurora kinases A and B in normal, hyperplastic, and malignant human endometrium: Aurora B as a predictor for poor prognosis in endometrial carcinoma. Hum Pathol 2005;36(12):1281-1288

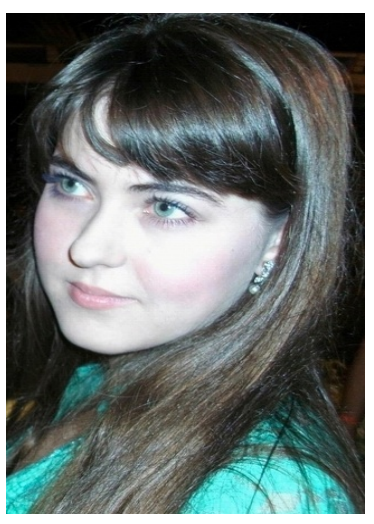

\section{Author Profile}

Liana Dedina is currently pursuing MSc degree in Pharmacology and Biomedical Toxicology at the University of Toronto. Her thesis project focuses on the expression of xenobiotic and nutrient transporters in the mammary gland, and potential implications for breastfeeding mother-infant pairs. Her research interests include (but are not limited to) toxicology and therapeutics, pregnancy and lactation, drug transport and metabolism, physiology and experimental medicine. 